\title{
Construction of a photovoltaic glucose sensor applying a metal-insulator-silicon structure in combination with ultra-thin polypyrrole-glucose oxidase film
}

\author{
J. Wang, H.X. Zhao, L.P. Du, H. Cai, P. Wang \\ Biosensor National Special Lab, Key Lab for Biomedical Engineering of Ministry of Education, \\ Department of Biomedical Engineering, Zheda Road No. 38, Zhejiang University, \\ Hangzhou 310027, China \\ Corresponding author's e-mail address: cnpwang@zju.edu.cn
}

\begin{abstract}
:
An enzyme-metal-insulator-silicon (EMIS) structured chemical sensor based on photovoltage technology is constructed for potentiometric biosensing of glucose in a flow-injection analysis (FIA) system. To enhance the sensibility of the sensor to glucose, an ultra-thin $(60 \mathrm{~nm})$ film of polypyrrole (PPy)-glucose oxidase (GOD) is formed as the glucose-sensitive membrane through a simple electropolymerization process in the absence of supporting electrolyte. The introduction of photovoltage technology and the electroimmobilization of GOD are advantageous in improving the sensitivity, selectivity, response time, linear range, stability and lifetime of potentiometric sensor for glucose measurement. Thus the photoelectric hybrid glucose sensor has extensive potential both in rapid determination of plasma glucose and long-time continuous monitoring of glucose concentration in extracellular microenvironment.
\end{abstract}

Key words: photovoltage technology, potentiometric biosensing, glucose sensor, flow-injection analysis, electropolymerization

\section{Introduction}

The detection of glucose has attracted most interest since the first publication on a glucose sensor [1]. There has been a constant increase in the number of studies devoted to glucose biosensing, among which amperometric technique is most widely used [2]. Although the amperometric glucose sensor has relatively high sensitivity and low detection limit, it seems to exhibit some disadvantages such as selectivity due to the interference of other reducible species in plasma. Besides, the electrocatalytic oxidation is not beneficial to the continuous monitoring of changes in glucose concentration. To overcome these drawbacks, an EMIS sensor based on photovoltage technology is constructed for rapid, long-time and continuous detection of glucose concentration.

Using the photovoltage technology employed in the light addressable potentiometric sensor [2], many biosensing devices are developed for enzyme based organic substances test $[3,4]$. The basic method lies in the $\mathrm{pH}$ variation induced by the enzymatic reactions as these biosensors are sensitive to $\mathrm{pH}$ change.
However, the $\mathrm{pH}$ deviating from the optimum $\mathrm{pH}$ value would affect the enzyme activity to a certain extent, which in turn will impact the sample detection. In view of this, an electrolytemetal-insulator-silicon structured sensor [5], which is sensitive to the redox potential determined by the ratio of oxidant and reductant in the analyte, has potential to overcome this problem.

Electropolymerized electroactive polymers have been widely used for the immobilization of enzymes in the preparation of biosensors because of the simplified technology of microbiosensor production. In this paper the potentiometric glucose sensor is developed by modifying the metal layer of the sensor electrochemically with an ultra-thin PPy-GOD film [6]. With the application of the photovoltage technology, high sensitivity to surface potential change and continuous electrostatic measurement can be realized.

\section{Experimental}

All chemicals were of analytical grade. Glucose oxidase from Aspergillus niger was obtained from Sigma. Pyrrole (from Aldrich) was distilled before use, stored in a refrigerator and 
protected from light. $\beta-D-G l u c o s e$ was prepared with $0.05 \mathrm{M}$ phosphate buffer $(\mathrm{pH} 7)$. All solutions were prepared with deionized water.

The potentiometric transducer is fabricated with the layer sequence of $\mathrm{Al} / \mathrm{n}-\mathrm{Si} / \mathrm{SiO}_{2} / \mathrm{Cr} / \mathrm{Au}$, as shown in Fig. 1(a). $50 \mathrm{~nm} \mathrm{SiO}_{2}$ is grown on a sample n-type silicon with a thickness of 100 $\mu \mathrm{m}$. Then $30 \mathrm{~nm} \mathrm{Cr}$ and $150 \mathrm{~nm} \mathrm{Au}$ are deposited on the $\mathrm{SiO}_{2}$ surface, followed by a lithography process to expose the working electrode and the lead wire. Finally $300 \mathrm{~nm} \mathrm{Al}$ is evaporated onto the rear side of the chip to form an ohmic contact.

The sensor surface is thoroughly cleaned before immobilization of GOD. Electropolymerization is carried out galvanostatically in stagnant solutions in a three-electrode cell with $\mathrm{Pt}$ auxiliary and $\mathrm{Ag} / \mathrm{AgCl}$ reference electrodes. The electrical charge is set as $25 \mathrm{mC} / \mathrm{cm}^{2}$ with a current density of $0.05 \mathrm{~mA} / \mathrm{cm}^{2}$. The ultra-thin film is formed using $0.1 \mathrm{M}$ pyrrole (Py) and $20 \mathrm{U} / \mathrm{ml}$ GOD. After electropolymerization, the sensor is rinsed thoroughly with deionized water to remove any loosely bound enzyme.
The measurement circuit and flow-injection analysis system is shown in Fig. 1(c). By using photovoltage technology, the surface potential of the sensor is transformed to a photocurrent that can be detected with an external circuit. The characteristic current-voltage (I-V) curve is $\mathrm{S}$ shaped, as shown in Fig. 1(b).

When the sensor surface contacts a glucose solution, an equilibrium surface potential is formed because of the enzyme-catalyzed reaction

glulcose $+\mathrm{O}_{2} \stackrel{\text { GOD }}{\longrightarrow}$ gluconic acid $+\mathrm{H}_{2} \mathrm{O}_{2}$

And the oxidation-reduction (redox) pairs of $\mathrm{O}_{2}$ and $\mathrm{H}_{2} \mathrm{O}_{2}$ will generate an equilibrium redox potential on the Au surface. Thus a stable I-V curve can be obtained. Different glucose solutions will produce different I-V curves, and the shift of I-V curves along the bias voltage $(\Delta \mathrm{V})$ reflects the glucose concentration change. In continuous monitoring, the bias voltage is usually set at a fixed value, and the photocurrent variation $(\Delta \mathrm{l})$ also reflects the glucose concentration change.

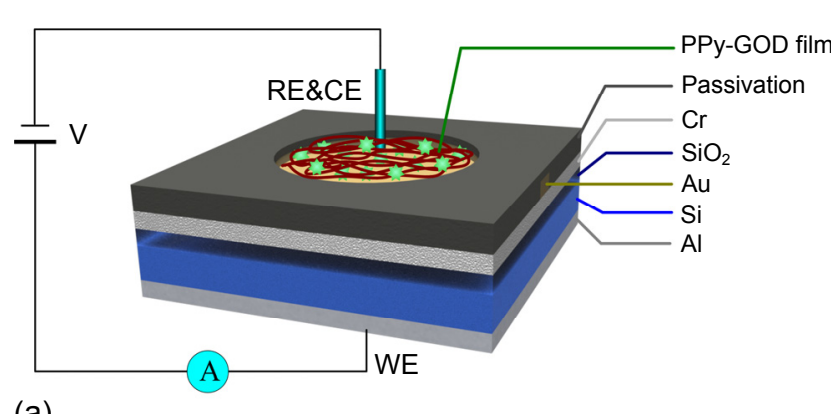

(a)
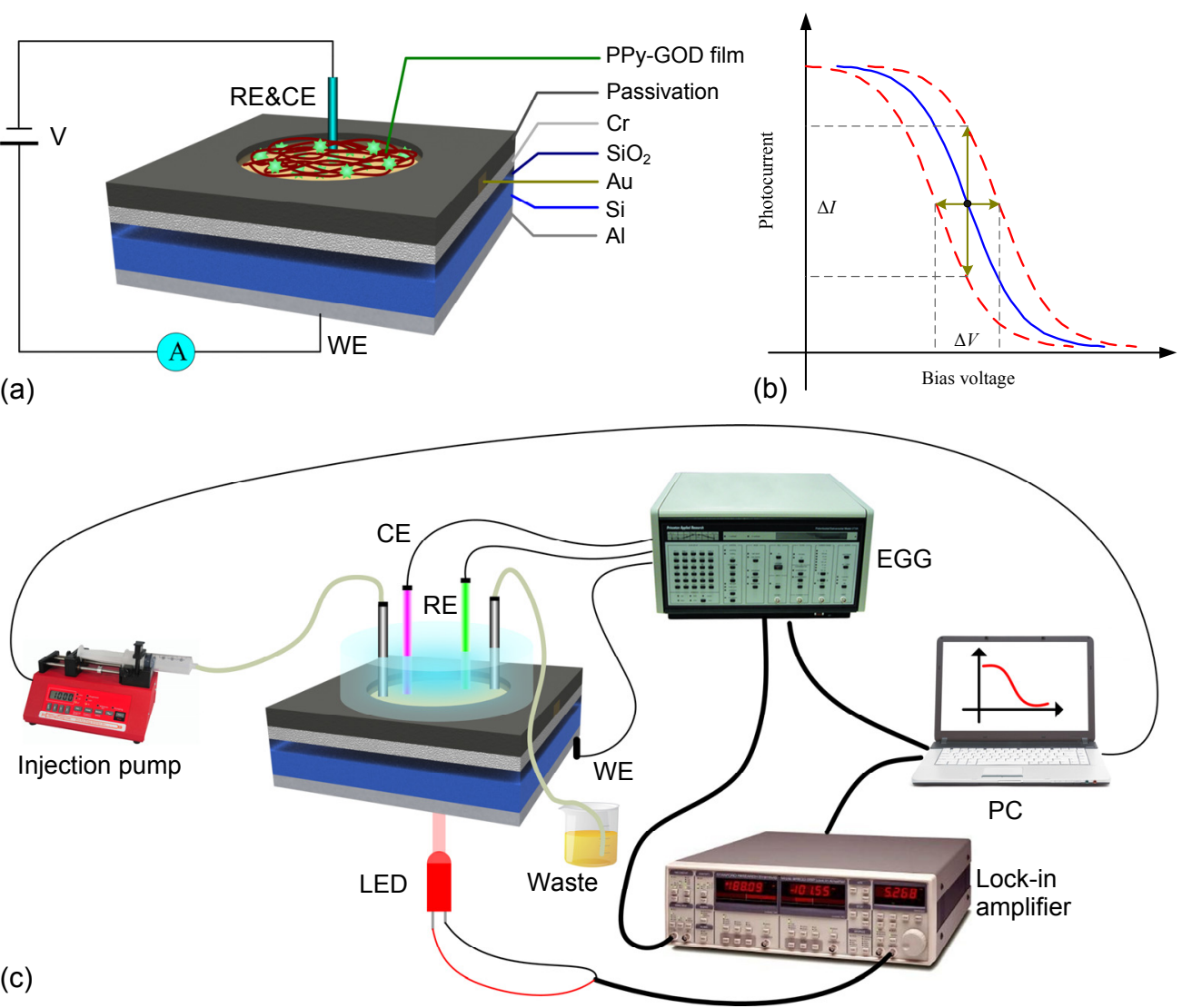

Fig. 1. Schematic diagram of experimental set up. (a) Sensor structure with sequence of $\mathrm{Al} / \mathrm{n}$-Si/SiO $\mathrm{O}_{2} / \mathrm{Cr} / \mathrm{Au} /$ PPy-GOD; (b) Characteristic curve of photovoltaic glucose sensor; (c) Measurement system and flow-injection analysis system. 


\section{Results and Discussion}

When in contact with glucose solution with different concentrations, the enzyme-catalyzed oxidation of glucose induces the surface potential change on the Au layer, which can be reflected through the I-V curve shift along the bias voltage. A potentiometric sensor is used for glucose detection immediately after electropolymerization. And the measurements are carried out in stagnant solutions. Fig. 2(a) shows the I-V curves of 5 glucose solutions with different concentrations $(0.04 \mathrm{mM}, 0.16 \mathrm{mM}$, $0.63 \mathrm{mM}, 2.51 \mathrm{mM}$ and $10 \mathrm{mM}$ ). The relationship between the bias voltage at the inflection point of I-V curve and the logarithm of glucose concentration is shown in Fig. 2(b). The sensitivity is $106.3 \mathrm{mV} / \mathrm{dec}$. Besides, the linear correlation coefficient is about 0.9963 , which means the bias voltage has good linear relationship with log(glucose concentration) within the range of about $40 \mu \mathrm{M} \sim 10 \mathrm{mM}$. The linear range of the ultra-thin PPy-GOD photovoltaic glucose sensor is significantly better than the range of $1 \sim 3 \mathrm{mM}$ and $1.5 \sim 10$ $\mathrm{mM}$ obtained in other studies.

(a)
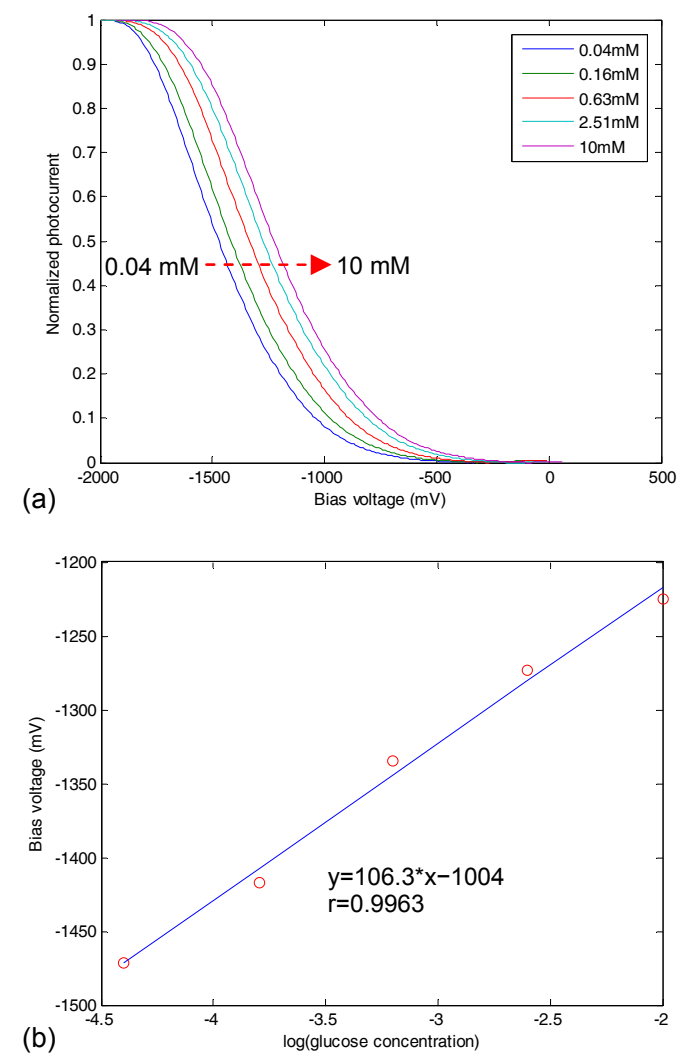

Fig. 2. Experimental result for glucose detection immediately after electropolymerization. (a) Normalized I-V curves for different glucose concentration. The legend shows the concentration; (b) Linear relationship between bias voltage and log(glucose concentration).
The result of continuous glucose monitoring is shown in Fig. 3. The bias voltage was fixed at $-1300 \mathrm{mV}$ in measurement. The responsive photocurrent shows a gradient curve for different glucose concentrations, which demonstrates the detection capability of the AuPPy-GOD film in the range of $40 \mu \mathrm{M} \sim 10 \mathrm{mM}$. The response time for glucose sensing is about 5 minutes. As the samples are measured in stagnant solutions, another 5 minutes are taken to obtain stable potentials, while a longer period of time is needed for high glucose concentrations. This may be due to the limited catalytic activity of the enzyme under the activity units of $20 \mathrm{U} / \mathrm{ml}$.

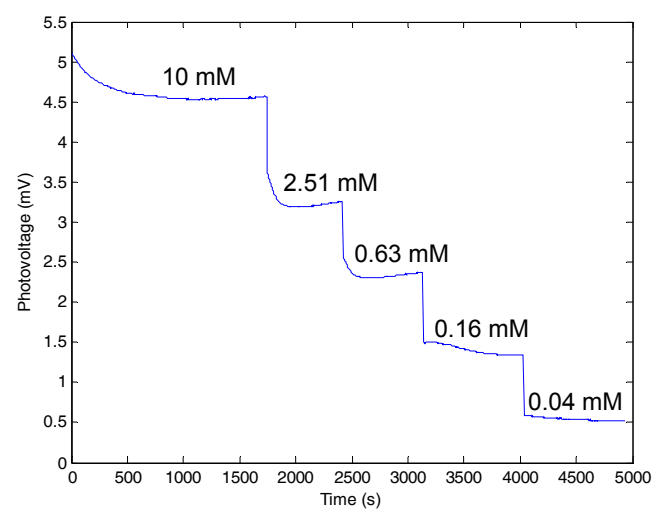

Fig. 3. Continuous monitoring of glucose concentration. The values of concentration are marked on the curve.

\section{Conclusions}

In this study a photovoltaic glucose sensor with an ultra-thin PPy-GOD film was developed. The $\mathrm{Au}$ layer of an EMIS structured sensor was modified with the PPy-GOD film through a simple electropolymerization process. The sensitive film Au-PPy-GOD showed good linearity and stability in the range of $40 \mu \mathrm{M} \sim 10$ $\mathrm{mM}$ with sensitivity of $106.3 \mathrm{mV} / \mathrm{dec}$. The photoelectric hybrid glucose sensor has extensive potential both in rapid determination of plasma glucose and long-time continuous monitoring of glucose concentration in extracellular microenvironment.

\section{Acknowledgements}

This work was supported by the National Natural Science Foundation of China (Grant No. $60725102,30970765,81027003)$ and the National Basic Research Program of China (Grant No. 2009CB320303). 


\section{References}

[1] L.C. Clarc, C. Lyons, Electrode Systems for Continuous Monitoring in Cardiovascular Surgery, Annals of the New York Academy of Sciences 102, 29-45 (1962); doi: 10.1111/j.17496632.1962.tb13623.x

[2] J. Wang, Electrochemical Glucose Biosensors, Chemical Reviews 108, 814-825 (2008); doi: 10.1021/cr068123a

[3] D.G. Hafeman, J.W. Parce, H.M. Mcconnell, Light-Addressable Potentiometric Sensor for Biochemical Systems Science 240, 1182-1185 (1988); doi: 10.1126/science.3375810

[4] J.C. Fernando, K.R. Rogers, N.A. Anis, J.J. Valdes, R.G. Thompson, A.T. Eldefrawi, M.E. Eldefrawi, Rapid Detection of Anticholinesterase Insecticides by a Reusable Light Addressable Potentiometric Biosensor, Journal of Agricultural and Food Chemistry 41, 511-516 (1993); doi: 10.1021/jf00027a031

[5] M. Shimizu, Y. Kanai, H. Uchida, T. Katsube, Integrated Biosensor Employing a Surface Photovoltage Technique, Sensors and Actuators $B$ 20, 187-192 (1994); doi: 10.1016/09254005(94)01176-1

[6] M. Adami, M. Martini, L. Piras, Characterization and Enzymatic Application of a Redox Potential Biosensor Based on a Silicon Transducer, Biosensors \& Bioelectronics 10, 633-638 (1995); doi: 10.1016/0956-5663(95)96939-V

[7] S.B. Adeloju, A.N. Moline, Fabrication of UltraThin Polypyrrole-Glucose Oxidase Film from Supporting Electrolyte-Free Monomer Solution for Potentiometric Biosensing of Glucose, Biosensors \& Bioelectronics 16, 133-139 (2001); doi: 10.1016/S0956-5663(00)00117-2 